\title{
EXCITED BARYONS IN A COVARIANT THREE-BODY BETHE-SALPETER APPROACH*
}

\author{
HÈLIOS SANCHIS-ALEPUZ ${ }^{\dagger}$ \\ Institut für Theoretische Physik, Justus-Liebig Universität Giessen, Giessen, Germany \\ Helios.Sanchis-Alepuz@theo.physik.uni-giessen.de \\ STANISLAV KUBRAK \\ Institut für Theoretische Physik, Justus-Liebig Universität Giessen, Giessen, Germany \\ Stanislav.D.Kubrak@physik.uni-giessen.de \\ CHRISTIAN FISCHER \\ Institut für Theoretische Physik, Justus-Liebig Universität Giessen, Giessen, Germany \\ Christian.Fischer@theo.physik.uni-giessen.de
}

Published 14 January 2014

\begin{abstract}
We present first results for the excited nucleon spectrum in the framework of Poincarécovariant three-body Bethe-Salpeter equations using the Rainbow-Ladder truncation of the interaction kernel. As expected, this truncation does not provide the mechanisms for a correct description of the spectrum. We also comment on possible steps beyond Rainbow-Ladder.
\end{abstract}

Keywords: Bethe-Salpeter; Nstar; Rainbow-Ladder.

PACS numbers: 12.38.Aw, 12.38.Lg, 14.40.Be, 14.20.Gk

\section{Introduction}

The continuum approach to hadron physics based on solutions of Dyson-Schwinger (DSE) and Bethe-Salpeter (BSE) equations (see e.g. Ref. and references therein) has as one of its main goals the description of hadron properties using the elementary degrees of freedom of QCD. However, the complications inherent to this enterprise have led to simplify this problem using the so-called Rainbow-Ladder (RL) truncation for the interaction kernels and vertices. This truncation is the simplest possible one preserving covariance and chiral symmetry. Moreover, this truncation describes

\footnotetext{
*This is an Open Access article published by World Scientific Publishing Company. It is distributed under the terms of the Creative Commons Attribution 3.0 (CC-BY) License. Further distribution of this work is permitted, provided the original work is properly cited.

†'Speaker.
} 
reasonably well a number of hadron observables, namely pseudoscalar ground-state meson masses and decay constants, ${ }^{1-3}$ ground-state baryon masses ${ }^{4,5}$ and form factors. ${ }^{6-10}$

The limitations of this very simple truncation have, nevertheless, been observed for example in the description of scalar and vector mesons, mesonic excited states ${ }^{11}$ or in the low-Q behavior of baryon electromagnetic form factors. ${ }^{8-10}$ In this work we report on the failure of the RL truncation to describe the excited-state spectrum of nucleons.

\section{Three-Body Bethe-Salpeter Equation Truncations}

The baryon masses and structure are obtained by solving the three-body BetheSalpeter equation

$$
\Psi=-i K G_{0} \Psi
$$

where $-i K$ is the three-quark interaction kernel, which includes three- and twoparticle irreducible interactions, and $G_{0}$ is the disconnected product of three full quark propagators. These propagators $S$ are given by the quark propagator DSE

$$
S^{-1}(p)=S_{0}^{-1}(p)+Z_{1 f} \int_{q} \gamma^{\mu} D_{\mu \nu}(p-q) \Gamma_{g q q}^{\nu}(p, q) S(q),
$$

where $S_{0}$ is the (renormalized) bare propagator, $\Gamma_{g q q}^{\nu}$ is the full quark-gluon vertex, $D^{\mu \nu}$ is the full gluon propagator and $Z_{1 f}$ and $Z_{2}$ are renormalization constants.

In this work we use the Rainbow-Ladder truncation, where for the quark-gluon vertex one uses a dressed tree-level vertex and the Bethe-Salpeter kernel is reduced to a dressed two-body vector-vector gluon exchange, neglecting three-body irreducible interactions. As a model for the dressing we use the Maris-Tandy model ${ }^{2,3}$

$$
\alpha_{\mathrm{eff}}\left(q^{2}\right)=\frac{\pi c}{\omega^{7}}\left(\frac{q^{2}}{\Lambda_{t}^{2}}\right)^{2} e^{-\frac{q^{2}}{\omega^{2} \Lambda_{t}^{2}}}+\frac{2 \pi \gamma_{m}\left(1-e^{-q^{2} / \Lambda_{t}^{2}}\right)}{\ln \left[e^{2}-1+\left(1+q^{2} / \Lambda_{Q C D}^{2}\right)^{2}\right]},
$$

which reproduces the one-loop QCD behavior in the UV and features a Gaussian distribution in the intermediate momentum region that provides dynamical chiral symmetry breaking. The scale $\Lambda_{t}=1 \mathrm{GeV}$ is introduced for dimensional reasons and has no impact on the results. Therefore, the interaction strength is characterized by the parameter $c$, fixed to $c=0.373$ to reproduce correctly the pion decay constant from the RL-truncated meson-BSE. The parameter $\omega$ controls the width of the interaction and is usually fixed to $\omega=0.4$. Ground-state masses are very often insensitive to the precise value of $\omega$. We analize in this work whether this is the case for baryon excited states as well. For the anomalous dimension we use $\gamma_{m}=$ $12 /\left(11 N_{C}-2 N_{f}\right)=12 / 25$, corresponding to $N_{f}=4$ flavors and $N_{c}=3$ colors. For the QCD scale $\Lambda_{Q C D}=0.234 \mathrm{GeV}$.

Equation (1) is usually solved as an eigenvalue problem by introducing a fictitious eigenvalue and using the baryon mass as a parameter. Those values of the baryon mass for which one obtains an eigenvalue equal to one correspond to a real 
bound-state. This procedure, however, may introduce many fictitious pairs of couple conjugate eigenvalues whose positions in the spectrum are not known beforehand and turn the numerical determination of the physical eigenvalues into too expensive a numerical task. An alternative method was used in Ref. 12 to study the spectrum of excited mesons. The idea is to modify Eq. (1) and transform it into an inhomogenoeus BSE for a vertex function $\Gamma$

$$
\Gamma=\Gamma_{0}-i K G_{0} \Gamma
$$

where the inhomogenous term $\Gamma_{0}$ is arbitrary and must have the appropriate quantum numbers. One then seeks for poles in the vertex function, which correspond to bound states. We use this last approach in the present work.

Finally, the choice of quantum numbers is done by expanding either $\Psi$ or $\Gamma$ in a suitable basis. ${ }^{4,5}$ To construct this basis only the requirements of Poincaré covariance and parity invariance are needed.

\section{Results}

In Table 1 we show our results for the masses of the nucleon ground states, with positive and negative parity, and for the first excited state of positive parity. We identify the negative parity ground state and the positive parity first excited state with the experimental states $N(1535)$ and $N(1440)$, respectively. At this stage of our calculations, however, these are the only states we are able to resolve because we use too low precision and the Bethe-Salpeter amplitudes of further states appear to be very oscillating. For this reason this identification might be wrong and these states might correspond to spurious (unphysical) solutions.

Nevertheless, assuming the identification of these solutions with physical states is correct, we have studied the dependence of the results with the interaction width $\omega$. For the central value $\omega=0.4$ the excited-state masses clearly disagree with the experimental values; if, as stated in Ref. 13, our results should be compared with the masses of bare resonances in dynamical coupled-channel approaches, the disagreement is even larger. Also, the ordering of negative- and positive-parity states is wrong.

As already pointed out in previous calculations, ${ }^{4}$ the positive-parity ground-state mass is insensitive to the value of $\omega$ around $\omega=0.4$. However, the mass of the first excited state is strongly dependent on this value, thus revealing an undesired model

Table 1. Masses of nucleon ground state, and first negative and positive excited states. The different rows correspond to different choices of the interaction width $\omega$, for constant integrated strength $c=0.373$.

\begin{tabular}{c|c|c|c|} 
& $\mathrm{N}(938)$ & $\mathrm{N}(1440) 1 / 2^{+}$ & $\mathrm{N}(1535) 1 / 2^{-}$ \\
\hline \hline$\omega=0.4$ & $0.93(5)$ & $1.20(4)$ & $1.12(4)$ \\
\hline$\omega=0.5$ & $0.94(5)$ & $1.03(5)$ & - \\
\hline$\omega=0.3$ & $0.94(5)$ & $1.47(5)$ & 1.07 \\
\hline
\end{tabular}


dependence. For the negative-parity state the $\omega$-dependence does not seem to be so important, although for $\omega=0.5$ we do not obtain any bound-state (possibly due to numerical unaccuracies). In all cases, the ordering of states is not changed and, in summary, the description of baryon excited-states within the Rainbow-Ladder truncation is not satisfactory.

\section{Summary}

We have performed the first calculation of excited nucleon masses in a three-body covariant Bethe-Salpeter framework. The main conclusion of our results, in agreement with previous calculations on the spectrum of excited mesons, is that the Rainbow-Ladder truncation does not contain the mechanisms for a correct description of excited states.

We are currently working on the simplest extension of these calculations beyond Rainbow-Ladder, namely, the inclusion of a Pion exchange in the two-body interaction kernel. In constituent quark models this term is enough to, at least, reverse the order of the negative- and positive-parity nucleon excited states.

\section{Acknowledgments}

This work was supported by the Helmholtz International Center for FAIR within the LOEWE program of the State of Hesse, the Helmholtz Young Investigator Group No. VH-NG-332, and by DFG TR16.

\section{References}

1. P. Maris, C. D. Roberts and P. C. Tandy, Phys. Lett. B 420 (1998) 267 [nuclth/9707003].

2. P. Maris and C. D. Roberts, Phys. Rev. C 56 (1997) 3369 [nucl-th/9708029].

3. P. Maris and P. C. Tandy, Phys. Rev. C 60 (1999) 055214 [nucl-th/9905056].

4. G. Eichmann, R. Alkofer, A. Krassnigg and D. Nicmorus, Phys. Rev. Lett. 104 (2010) 201601 [arXiv:0912.2246 [hep-ph]].

5. H. Sanchis-Alepuz, G. Eichmann, S. Villalba-Chavez and R. Alkofer, Phys. Rev. D 84 (2011) 096003 [arXiv:1109.0199 [hep-ph]].

6. P. Maris and P. C. Tandy, Phys. Rev. C 62 (2000) 055204 [nucl-th/0005015].

7. P. Maris and P. C. Tandy, Phys. Rev. C 65 (2002) 045211 [nucl-th/0201017].

8. G. Eichmann, Phys. Rev. D 84 (2011) 014014 [arXiv:1104.4505 [hep-ph]].

9. G. Eichmann and D. Nicmorus, Phys. Rev. D 85 (2012) 093004 [arXiv:1112.2232 [hep-ph]].

10. H. Sanchis-Alepuz, R. Williams and R. Alkofer, Phys. Rev. D 87 (2013) 095015 [arXiv:1302.6048 [hep-ph]].

11. A. Krassnigg and M. Blank, Phys. Rev. D 83 (2011) 096006 [arXiv:1011.6650 [hep$\mathrm{ph}]]$.

12. M. Blank and A. Krassnigg, Comput. Phys. Commun. 182 (2011) 1391 [arXiv:1009.1535 [hep-ph]].

13. H. L. L. Roberts, L. Chang, I. C. Cloet and C. D. Roberts, Few Body Syst. 51 (2011) [arXiv:1101.4244 [nucl-th]]. 